\title{
Differential pain response at local and remote muscle sites following aerobic cycling exercise at mild and moderate intensity
}

\author{
Peter S. Micalos ${ }^{1 *}$ (D) and Lars Arendt-Nielsen²
}

\begin{abstract}
Physical exercise has been shown to inhibit experimental pain response in the post-exercise period. Modulation of the pain system may be differentiated between muscle sites engaging in contractile activity. The purpose of this study was to assess the pain response at remote and local muscle sites following aerobic exercise at different work intensities. Participants included 10 healthy and physically active males (mean age \pm SD, $21.2 \pm 3.4$ ). Somatic pressure pain threshold (PPT) at the rectus femoris (local) and brachioradialis (remote) muscle site was measured at before (Pre), 5 min after (Post1), and 15 min after (Post2) aerobic cycling exercise at 70 and $30 \%$ of peak oxygen uptake $\left(\mathrm{VO}_{2 \text { peak }}\right)$ performed on different occasions in a counterbalanced order, separated by minimum of 3 days interval. Repeated measures ANOVA for PPT reveals significant main effect for time $(f=3.581, p=0.049$, observed power $=0.588)$ and muscle site $(f=17.931, p=0.002$, observed power $=0.963)$. There was a significant interaction shown for exercise intensity by time $(f=11.390, p=0.012$, observed power $=0.790)$. PPT at rectus femoris following cycling exercise at $70 \%$ of $\mathrm{VO}_{2 \text { peak }}$ reveals a significant increase between Pre-Post1 $(p=0.040)$. PPT for rectus femoris following cycling exercise at $30 \%$ of $\mathrm{VO}_{2 \text { peak }}$ revealed a significant decrease between Pre-Post1 $(p=0.026)$ and Pre-Post2 $(p=0.008)$.

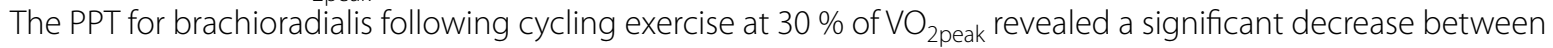
Pre-Post1 ( $p=0.011)$ and Pre-Post2 $(p=0.005)$. These results show that aerobic exercise increases PPT locally at the exercise muscle site following exercise at $70 \%$ of $\mathrm{VO}_{2 \text { peak }}$ but reduces PPT following exercise at $30 \%$ of $\mathrm{VO}_{2 \text { peak }}$.
\end{abstract}

Keywords: Hypoalgesia, Cycling exercise, Peak oxygen uptake, Pressure pain threshold

\section{Background}

Physical exercise has been shown to modulate experimental pain response in the post-exercise period (O'Connor and Cook 1999). Aerobic exercise such as cycling and running has been shown to reduce the sensitivity to experimental pain after exercise which returns to baseline within $30 \mathrm{~min}$ (Hoffman et al. 2004). The level of exercise to elicit pain inhibition in the post exercise period requires an intensity of $60-75 \%$ of maximum oxygen consumption (Koltyn 2002) or at $50 \%$ of heart rate (HR) reserve (Naugle et al. 2014). Hypoalgesic effects have been shown with exercise duration of $30 \mathrm{~min}$ but not at 10 min (Hoffman et al. 2004). Research on the

\footnotetext{
*Correspondence: pmicalos@csu.edu.au

1 School of Biomedical Sciences, Charles Sturt University, Panorama Ave,

Bathurst, NSW 2795, Australia

Full list of author information is available at the end of the article
}

effect of exercise on pain response is of interest due to evaluating changes in the function of the pain inhibitory system (Micalos et al. 2014). Understanding the function of the pain inhibitory system is beneficial for improving the prescription of exercise in chronic pain therapy (Carbonell-Baeza et al. 2010).

Experimental pain assessment requires the application of a controlled noxious stimulus to evaluate pain responses before and after intervention such as physical exercise. Experimental pain stimuli such as somatic mechanical pressure are applied in pain research settings to assess the function of the pain system (Kosek and Lundberg 2003). The pressure pain threshold (PPT) is the minimum required somatic stimulus intensity applied on the body surface to evoke a pain response (Fischer 1987; Cathcart and Pritchard 2006; Walton et al. 2011). Assessment of PPT involves application of an increasing

\section{黛 Springer}


pressure stimulus on the skin by an algometer until the signal for pain is conveyed (Ohrbach and Gale 1989). Previous research indicates that aerobic exercise reduces pain sensitivity (Hoffman et al. 2004) and increases the PPT after exercise (Koltyn et al. 1996) in healthy participants.

The mechanism underlying the exercise-induced pain inhibition has not been fully elucidated (Koltyn et al. 2014). It has been shown that the hypoalgesia following exercise is evident throughout the body and regionally at the exercise muscle site (Kosek and Lundberg 2003). This suggests that physical exercise activates central descending pathways for widespread pain inhibition, however, this effect is also augmented by localised pain inhibitory mechanisms at the exercising muscle site. In support of this, it has been shown that aerobic exercise increases the PPT to a greater extent at the site of the exercising body part (Vaegter et al. 2014). However, the effect of different exercise intensities has not been fully elucidated together with regional pain inhibition. Therefore, the purpose of this study is to assess the pain threshold at local and remote muscle sites following aerobic exercise at different intensities to investigate the balance between regional and widespread somatic pain sensitivity.

\section{Results}

Mean age, height, body weight, and $\mathrm{VO}_{2 \text { peak }}( \pm \mathrm{SD})$ of participants are shown in Table 1 . Mean power output $( \pm \mathrm{SD})$ for submaximal exercise at 70 and $30 \%$ of $\mathrm{VO}_{2 \text { peak }}$ was $186 \pm 24$ and $84.5 \pm 15.4 \mathrm{~W}$, respectively. The mean time interval ( \pm SD) between the sessions was $6.3 \pm 3.3$ days. The mean HR and RPE response at $70 \%$ $\mathrm{VO}_{2 \text { peak }}$ was $151.8 \pm 16.7$ beats/min and $13.5 \pm 1.3$ units, respectively. At $30 \% \mathrm{VO}_{2 \text { peak }}$ the mean $\mathrm{HR}$ and RPE was $106.4 \pm 20.4$ beats/min and RPE $9.9 \pm 3.2$ units.

Results for Pre-exercise intra-class correlation coefficient show 0.63 for brachioradialis and 0.67 for rectus femoris muscles, respectively. Repeated measures ANOVA for PPT reveals significant main effect for time ( $\mathrm{f}=3.581$, $p=0.049$, observed power $=0.588$ ) and muscle site ( $\mathrm{f}=17.931, p=0.002$, observed power $=0.963$ ). There was a significant interaction shown for exercise intensity by time $(f=11.390, p=0.012$, observed power $=0.790)$.

Based on results for main effects, separate analyses were performed for exercise intensity. The mean PPT at the rectus femoris and brachioradialis sites following aerobic exercise at $70 \%$ of $\mathrm{VO}_{2 \text { peak }}$ are shown in Fig. 1a. $\mathrm{PPT}$ at rectus femoris following cycling exercise at $70 \%$

\section{Table 1 Participant characteristics}

\begin{tabular}{lccc}
\hline Age (years) & Height $(\mathbf{c m})$ & Mass $(\mathbf{k g})$ & VO $_{\text {2peak }}(\mathbf{I} / \mathbf{m i n})$ \\
\hline $21.2( \pm 3.4)$ & $179.5( \pm 5.8)$ & $77.1( \pm 9.3)$ & $3.5( \pm 0.4)$ \\
\hline
\end{tabular}

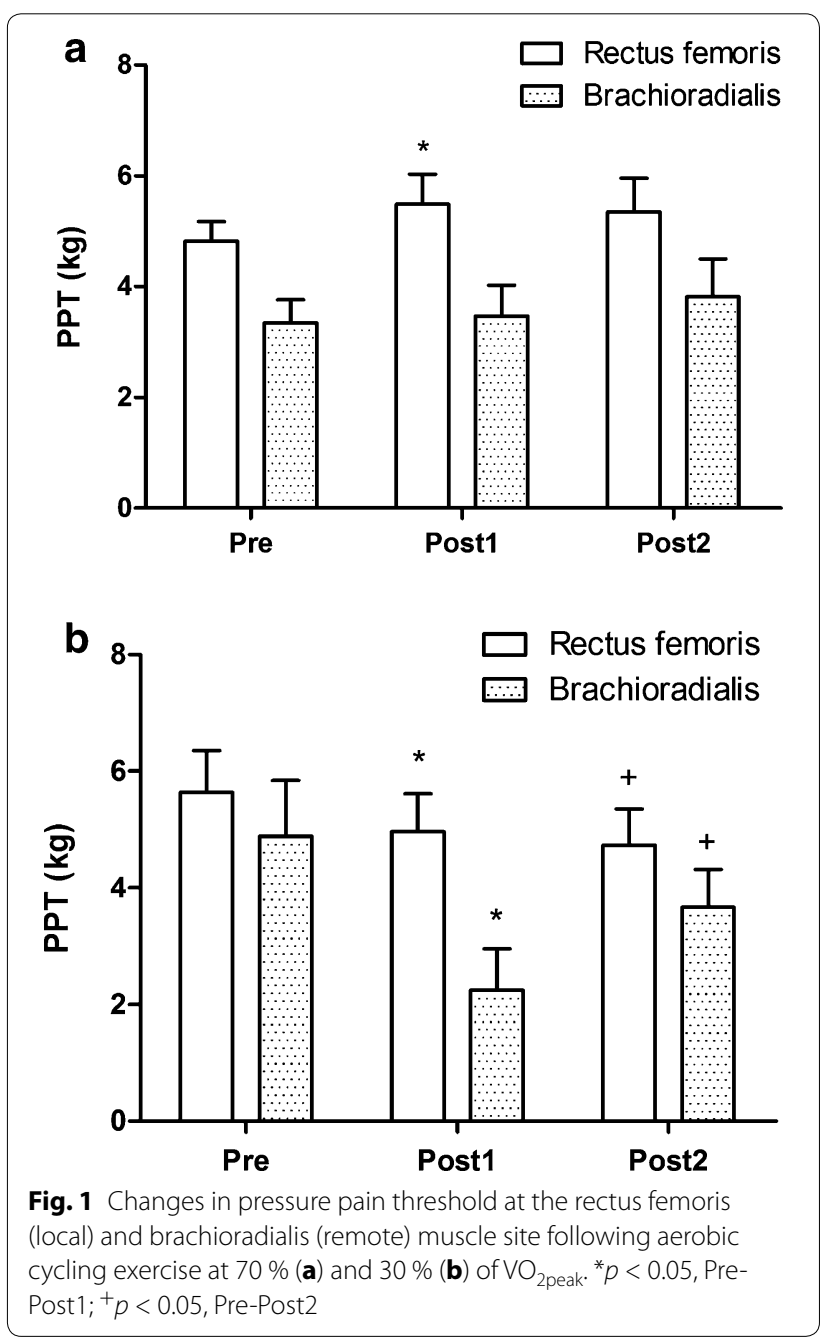

of $\mathrm{VO}_{2 \text { peak }}$ reveals a significant increase between PrePost1 $(p=0.040)$ but not for Pre-Post2 $(p=0.131)$. The PPT for brachioradialis following cycling exercise at $70 \%$ of $\mathrm{VO}_{2 \text { peak }}$ reveals no significant difference between PrePost1 and Pre-Post2.

The PPT following aerobic exercise at $30 \%$ of $\mathrm{VO}_{2 \text { peak }}$ at the rectus femoris and brachioradialis muscle sites are shown in Fig. 1b. PPT for rectus femoris following cycling exercise at $30 \%$ of $\mathrm{VO}_{2 \text { peak }}$ reveals a significant decrease between Pre-Post1 $(p=0.026)$ and Pre-Post2 $(p=0.008)$. The PPT for brachioradialis following cycling exercise at $30 \%$ of $\mathrm{VO}_{2 \text { peak }}$ reveals a significant decrease between Pre-Post1 $(p=0.011)$ and Pre-Post2 $(p=0.005)$.

\section{Discussion}

The present results reveal an increase in PPT at the local exercise muscle site following cycling exercise at $70 \%$ of peak oxygen uptake. Our results comply with previous research showing that aerobic exercise induces 
hypoalgesic effects at the exercising muscle site compared to the non-exercise site (Vaegter et al. 2014). These results are also in accord with previous research showing increased PPT following aerobic exercise at high intensity compared to low intensity exercise (Vaegter et al. 2014). However, the present results did not show a significant increase in PPT in the non-exercising muscle site following aerobic exercise at $70 \%$ of peak oxygen uptake. This level of exercise intensity was selected in the present study to enable participants to complete $30 \mathrm{~min}$ of exercise and show experimental pain inhibition (Koltyn 2002; Hoffman et al. 2004). A recent study showed moderate exercise intensity was insufficient to increase the PPT at the non-exercise site but was adequate following vigorous intensity exercise (Naugle et al. 2014). Hence, the aerobic exercise at $70 \%$ of peak oxygen uptake in the present study was below a vigorous intensity to induce widespread pain inhibition. Additionally, the increase in PPT was observed immediately after exercise but was not significant at 15 min post-exercise. Together, this suggests moderate exercise intensity induces a transient localised pain inhibition at the exercising muscle site but not for widespread pain inhibition at the non-exercising muscle site.

Potential mechanisms for increased pain threshold at the exercising muscle site are associated with peripheral inhibition of the nociceptive signal. Endogenous opioid-related substances are expressed at central sites in the nervous system but are also locally expressed at the exercising muscle site and this may attenuate the nociceptive signal (Tegeder et al. 2003). However, caution must be applied when appraising exercise-induced pain inhibition with experimental stimuli. It has been shown that noxious thermal sensitivity such as cold and heat are not consistently inhibited following aerobic exercise (Ruble et al. 2005; Janal et al. 1984). In another study, reduced pressure pain sensitivity was observed following isometric exercise but not for aerobic exercise (Vaegter et al. 2015). Moreover, heat pain threshold was shown to be increased but not PPT following aerobic exercise (Kodesh and Weissman-Fogel 2014). Reduced pain sensitivity following exercise has been more consistently evident with suprathreshold noxious stimuli (Kodesh and Weissman-Fogel 2014; Naugle et al. 2012). Together, this suggests that the reduced pain sensitivity following exercise may be observed according to the type of noxious stimulus and the modality of exercise applied (Naugle et al. 2012; Lau et al. 2015). Despite these limitations, our results show an increase in the PPT at the exercising muscle site following moderate intensity aerobic exercise.

The present results also revealed a decrease in PPT following mild exercise at $30 \%$ of $\mathrm{VO}_{2 \text { peak. }}$. This mild exercise intensity was selected to enable a substantial difference in exercise responses between the moderate and mild intensity. The reduced PPT following exercise was unexpected, however, this has previously been demonstrated. In research by Lau et al. (2015), this shows a reduced PPT immediately after eccentric exercise. In contrast, no difference in pressure pain sensitivity following low intensity aerobic exercise at $50 \%$ of maximum oxygen uptake has been reported (Vaegter et al. 2014; Hoffman et al. 2004). Reason for this disparity might be associated with the reduced exercise intensity in the present study which was set at $30 \%$ of $\mathrm{VO}_{2 \text { peak }}$. It is possible that mild exercise intensity may facilitate the somatosensory system and increase the sensitivity to noxious stimuli. Primary excitatory and inhibitory neurotransmitters in the central nervous system include glutamate and GABA, respectively. Several neurotransmitter substances have been shown to be centrally (Meeusen and De Meirleir 1995) and peripherally (Karlsson et al. 2015) released during exercise which can facilitate or inhibit pain signalling. In the animal model, enhanced GABA has been observed in the forebrain with regular exercise (Hill et al. 2008). The midbrain periaqueductal grey (PAG) and the rostral ventromedial medulla (RVM) have been shown to exert bidirectional facilitation and inhibition over nociceptive dorsal horn transmission (Fields et al. 2006). Using this paradigm, a threshold exercise stress potentially modulates central pain transmission by activating the PAGRVM network (Micalos 2014). Activation of this network is associated with the central endogenous release of neurotransmitters including opioids, serotonin, and cannabinoids (Fields et al. 2006). However, it is possible that mild aerobic exercise may facilitate ascending afferent signalling compared to moderate intensity exercise which activates the descending pain inhibitory system. Additional research would be required to further elucidate these changes in pain sensitivity following exercise at different intensities.

Several limitations in this study need to be acknowledged. Firstly the present study did not include a separate non-exercise control group. Additionally, the intra-class correlation analysis between trials at baseline showed moderate levels of reliability compared to previous research showing moderate to excellent reliability for PPT (Walton et al. 2011). This previous research used three repeated assessments of PPT at each site under resting conditions whereas the present study included a single assessment at bilateral muscle sites. However, due to differences between trials we analysed data separately for each exercise intensity. The data extracted for the present results were part of a larger data set involving noxious stimuli, however, the counterbalanced testing would have offset any cross-over effects. It should be noted that the transient pain inhibition limits the capacity to 
perform several repeated pain threshold assessments following exercise. Moreover, the participant sample may not generalise to older participants, females, and chronic disease patients.

\section{Conclusion}

In conclusion, these results show that aerobic activity attenuates pressure pain sensitivity locally at the exercise muscle site following cycling exercise at $70 \%$ of peak oxygen uptake, however, may facilitate pain sensitivity following exercise at $30 \%$ ofVO $\mathrm{P}_{2 \text { peak }}$. This indicates that localised pressure pain inhibition is shown at the exercise muscle site following moderate exercise but not for widespread pain inhibition.

\section{Methods}

The study was conducted with the approval of the Charles Sturt University Ethics in Human Research Committee (Approval Number: 2007/107) and all participants signed a letter of informed consent. The data extracted in the present study is a separate part of a larger data set that has been previously published (Micalos et al. 2015).

\section{Participants}

Participants were recruited from a university student population (Fig. 2). Participants included ten healthy and physically active males. Exclusion criteria included the recent use of anti-inflammatory and analgesic medications, lower limb injury or surgery, acute infection, and chronic disease.

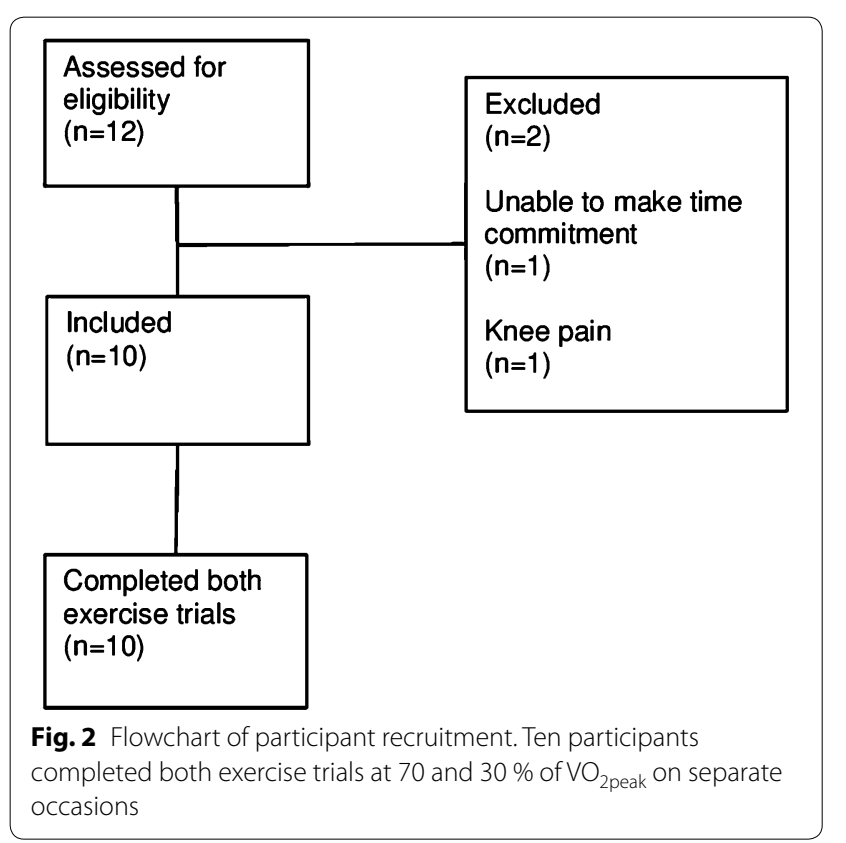

\section{Experimental design}

We used a mixed design repeated measures. Each participant attended the laboratory on three separate visitations. The first visitation was required to measure anthropometric data and assess peak oxygen uptake $\left(\mathrm{VO}_{2 \text { peak }}\right)$. A second and third visitation was required to assess PPT before and after cycling exercise intensity at 70 and $30 \%$ of $\mathrm{VO}_{2 \text { peak }}$, on separate visitations. A minimum of 3 days interval between trials was conducted to avert delayed exercise muscle soreness. The succession of exercise intensities was counterbalanced to avert an order effect. Participants were instructed to maintain their regular daily activities and to avoid exercise on the day when the experimental sessions were performed.

\section{Assessment of peak oxygen consumption}

The assessment of peak oxygen consumption was performed by ramped cycle exercise test (Jones et al. 2014). Peak exercise oxygen uptake was assessed by respiratory gas analyser (Par Medics, Pneumotach TrueOne 2400, Sandy, UT, USA) connected to a Hans Rudolph valve (Hans Rudolph Inc., Shawnee, KS, USA) during exercise on a stationary cycle ergometer (Lode, Sport Excalibur, Groningen, The Netherlands). The $\mathrm{VO}_{2 \text { peak }}$ assessment began at $60 \mathrm{~W}$ of power output and was incremented by $30 \mathrm{~W}$ at $3 \mathrm{~min}$ intervals until exercise tolerance. The HR was assessed by telemetry (Polar RS200, Polar Electro, Kempele, Finland).

\section{Assessment of pressure pain threshold}

The PPT was assessed before and after cycling exercise performed at 70 and $30 \%$ of $\mathrm{VO}_{2 \text { peak }}$ on two separate visitations in a counterbalanced order. PPT was assessed using a handheld pressure algometer (Force One, Wagner, USA) with a surface contact area of $1 \mathrm{~cm}^{2}$. The PPT was assessed by increasing the surface contact pressure until the participant verbally indicated that the stimulus was painful. All PPT assessments were performed by one experimenter over the muscle belly at the left and right rectus femoris (local) and brachioradialis (remote) muscle sites. Each participant was familiarised with the procedure prior to assessment of the PPT.

The PPT was assessed at before exercise (Pre), $5 \mathrm{~min}$ after exercise (Post1), and $15 \mathrm{~min}$ after exercise (Post2). Participants were seated in a reclined liftback table for the PPT assessments. Each aerobic exercise session was performed in a climate controlled laboratory at the same time of day to minimise diurnal variation. The duration of aerobic exercise was for $30 \mathrm{~min}$ on a bicycle (Avanti Bikes, Auckland, New Zealand) mounted on a stationary cycle trainer (Tacx Cycleforce Basic, Rotterdam, The Netherlands) with a power output display (SRM, Colorado Springs, USA). The exercise load required to maintain the designated power output was pre-determined at 
70 or $30 \%$ of $\mathrm{VO}_{2 \text { peak }}$ as shown by the power output display unit. The HR and rating of perceived exertion (RPE) (Borg 1970) were assessed every 2 min during exercise.

\section{Statistical analysis}

Intra-class correlation coefficient was used to determine PPT reliability for Pre-exercise at 70 and $30 \%$ of $\mathrm{VO}_{2 \text { peak }}$. Three-way repeated measures ANOVA with observed power analysis on PPTs were performed for exercise intensity $\left(70 \% \mathrm{VO}_{2 \text { peak }} ; 30 \% \mathrm{VO}_{2 \text { peak }}\right)$, muscle site (rectus femoris; brachioradialis) and time (Pre-Post1; Pre-Post2). Checks for data sphericity were performed and Greenhouse-Geisser procedure integrated where required. Where significant main effects were shown, paired samples statistics were performed on PPT's at 70 and $30 \% \mathrm{VO}_{2 \text { peak }}$ between Pre-Post1 and Pre-Post2. Data were analysed using IBM SPSS statistics v20. Results for PPT are presented as mean with standard error. Level of significance was set at $p<0.05$.

\section{Abbreviations}

HR: heart rate; PPT: pressure pain threshold; RPE: rating of perceived exertion; $\mathrm{VO}_{2 \text { peak: }}$ : peak oxygen uptake; W: Watt.

\section{Authors' contributions}

PM: Carried out substantial contributions to conception and design, acquisition of data, analysis and interpretation of data; drafting the manuscript. LA-N: revising manuscript critically for important intellectual content; final approval of the manuscript. Both authors read and approved the final manuscript.

\section{Author details}

1 School of Biomedical Sciences, Charles Sturt University, Panorama Ave, Bathurst, NSW 2795, Australia. ${ }^{2}$ Department for Health Sciences and Technology, Center for Sensory-Motor Interaction, School of Medicine, Aalborg University, Fredrik Bajers Vej 7-D3, Bld. D3, 9220 Ålborg, Denmark.

\section{Acknowledgements}

This research was supported by the Charles Sturt University-Faculty seed grant scheme.

\section{Competing interests}

The authors declare that they have no competing interests.

Received: 11 September 2015 Accepted: 14 January 2016 Published online: 28 January 2016

\section{References}

Borg G (1970) Perceived exertion as an indicator of somatic stress. Scand J Rehabil Med 2:92-98

Carbonell-Baeza A, Aparicio VA, Ortega FB, Cuevas AM, Alvarez IC, Ruiz JR, Delgado-Fernandez M (2010) Does a 3-month multidisciplinary intervention improve pain, body composition and physical fitness in women with fibromyalgia? Br J Sports Med 45(15):1189-1195

Cathcart S, Pritchard D (2006) Reliability of pain threshold measurement in young adults. J Headache Pain 7(1):21-26

Fields HL, Basbaum Al, Heinricher MM (2006) Central nervous system mechanisms of pain modulation. In: McMahon SB, Koltzenburg M (eds) Wall and Melzack's textbook of pain, 5th edn. Elsevier, Edinburgh, pp 125-142

Fischer AA (1987) Pressure algometry over normal muscles. Standard values, validity and reproducibility of pressure threshold. Pain 30(1):115-126

Hill LE, Droste SK, Nutt DJ, Linthorst ACE, Reul JMHM (2008) Voluntary exercise alters GABAA receptor subunit and glutamic acid decarboxylase-67 gene expression in the rat forebrain. J Psychopharmacol. doi:10.1177/0269881108096983

Hoffman MD, Shepanski MA, Ruble SB, Valic Z, Buckwalter JB, Clifford PS (2004) Intensity and duration threshold for aerobic exercise-induced analgesia to pressure pain. Arch Phys Med Rehabil 85(7):1183-1187

Janal MN, Colt EW, Clark WC, Glusman M (1984) Pain sensitivity, mood and plasma endocrine levels in man following long-distance running: effects of naloxone. Pain 19(1):13-25

Jones MD, Booth J, Taylor JL, Barry BK (2014) Aerobic training increases pain tolerance in healthy individuals. Med Sci Sports Exerc 46(8):1640-1647

Karlsson L, Gerdle B, Ghafouri B, Bäckryd E, Olausson P, Ghafouri N, Larsson B (2015) Intramuscular pain modulatory substances before and after exercise in women with chronic neck pain. Eur J Pain 19(8):1075-1085. doi:10.1002/ejp.630

Kodesh E, Weissman-Fogel I (2014) Exercise-induced hypoalgesia-interval versus continuous mode. Appl Physiol Nutr Metab 39(7):829-834. doi:10.1139/apnm-2013-0481

Koltyn KF (2002) Exercise-induced hypoalgesia and intensity of exercise. Sports Med 32(8):477-487

Koltyn KF, Garvin AW, Gardiner RL, Nelson TF (1996) Perception of pain following aerobic exercise. Med Sci Sports Exerc 28(11):1418-1421

Koltyn KF, Brellenthin AG, Cook DB, Sehgal N, Hillard C (2014) Mechanisms of exercise-induced hypoalgesia. J Pain 15(12):1294-1304. doi:10.1016/j. jpain.2014.09.006

Kosek E, Lundberg L (2003) Segmental and plurisegmental modulation of pressure pain thresholds during static muscle contractions in healthy individuals. Eur J Pain 7:251-258

Lau W, Blazevich A, Newton M, Wu S, Nosaka K (2015) Changes in electrical pain threshold of fascia and muscle after initial and secondary bouts of elbow flexor eccentric exercise. Eur J Appl Physiol 115(5):959-968. doi:10.1007/s00421-014-3077-5

Meeusen R, De Meirleir K (1995) Exercise and brain neurotransmission. Sports Med 20(3):160-188. doi:10.2165/00007256-199520030-00004

Micalos PS (2014) Perspectives on biochemical and neurosensory mechanisms for exercise-induced pain inhibition. Fatigue Biomed Health Behav 2(4):219-230. doi:10.1080/21641846.2014.957484

Micalos PS, Korgaonkar MS, Drinkwater EJ, Cannon J, Marino FE (2014) Cerebral responses to innocuous somatic pressure stimulation following aerobic exercise rehabilitation in chronic pain patients: a functional magnetic resonance imaging study. Int J Gen Med 7:425-432

Micalos PS, Harris J, Drinkwater EJ, Cannon J, Marino FE (2015) Perceptual and cerebro-spinal responses to graded innocuous and noxious stimuli following aerobic exercise. J Sports Med Phys Fit 55(11):1407-1415

Naugle KM, Fillingim RB, Riley JL III (2012) A meta-analytic review of the hypoalgesic effects of exercise. J Pain 13(12):1139-1150. doi:10.1016/j. jpain.2012.09.006

Naugle KM, Naugle KE, Fillingim RB, Samuels B, Riley JL 3rd (2014) Intensity thresholds for aerobic exercise-induced hypoalgesia. Med Sci Sports Exerc 46(4):817-825. doi:10.1249/mss.0000000000000143

O'Connor PJ, Cook DB (1999) Exercise and pain: the neurobiology, measurement, and laboratory study of pain in relation to exercise in humans. Exerc Sport Sci Rev 27:119-166

Ohrbach R, Gale EN (1989) Pressure pain thresholds in normal muscles: reliability, measurement effects, and topographic differences. Pain 37(3):257-263

Ruble SB, Hoffman MD, Shepanski MA, Valic Z, Buckwalter JB, Clifford PS (2005) Thermal pain perception after aerobic exercise. Arch Phys Med Rehabil 86(5):1019-1023

Tegeder I, Meier S, Burian M, Schmidt H, Geisslinger G, Lötsch J (2003) Peripheral opioid analgesia in experimental human pain models. Brain 126(5):1092-1102. doi:10.1093/brain/awg115

Vaegter HB, Handberg G, Graven-Nielsen T (2014) Similarities between exercise-induced hypoalgesia and conditioned pain modulation in humans. Pain 155(1):158-167. doi:10.1016/j.pain.2013.09.023

Vaegter HB, Handberg G, Graven-Nielsen T (2015) Isometric exercises reduce temporal summation of pressure pain in humans. Eur J Pain 17(7):973983. doi:10.1002/ejp.623

Walton D, MacDermid J, Nielsen W, Teasell R, Chiasson M, Brown L (2011) Reliability, standard error, and minimum detectable change of clinical pressure pain threshold testing in people with and without acute neck pain. J Orthop Sports Phys Ther 41(9):644-650. doi:10.2519/jospt.2011.3666 